Revue d'histoire de l'Amérique française

FBS REVUE D.HISTOIRE DE L'AMÉRIQUE FRANÇAISE

\title{
Les Canadiens français jugés par les Français de France, 1830-1939
}

\section{Armand Yon}

Volume 18, numéro 3, décembre 1964

URI : https://id.erudit.org/iderudit/302386ar

DOI : https://doi.org/10.7202/302386ar

Aller au sommaire du numéro

Éditeur(s)

Institut d'histoire de l'Amérique française

ISSN

0035-2357 (imprimé)

1492-1383 (numérique)

Découvrir la revue

Citer cet article

Yon, A. (1964). Les Canadiens français jugés par les Français de France, 1830-1939. Revue d'histoire de l'Amérique française, 18(3), 321-342.

https://doi.org/10.7202/302386ar d'utilisation que vous pouvez consulter en ligne.

https://apropos.erudit.org/fr/usagers/politique-dutilisation/ 


\section{Un siècle d'opinion française}

\section{LES CANADIENS FRANÇAIS JUGÉS PAR LES FRANÇAIS DE FRANCE \\ 1830-1939}

Au cours de longues années qu'il vécut en Europe, et particulièrement à Paris, M. l'abbé Armand Yon s'est plu à recueillir les témoignages de Français qui, à diverses époques, visitèrent notre pays.

Dans la première partie d'un ouvrage en préparation, intitulée "Les peintres de la vie canadienne", il passe en revue et juge ses témoins, quitte, dans une seconde partie, à nous livrer le "tableau" qui résulte de ces nombreuses appréciations.

De cet important travail, la Revue est heureuse de publier aujourd'hui un premier extrait, qui sera suivi de plusieurs autres.

Ire partie

\section{LES PEINTRES DE LA VIE CANADIENNE}

I. L'ĖRE DU SENTIMENT (1830-1880)

$\S$ 1. Pénétration lente (1830-1850)

Que savent du Canada les Français de la Restauration?

A-t-on toujours, en France, comme l'affirmait vers 1781 l'éditeur de Pouchot, “des idées si fausses de ce pays, qu'on y réduit toute son utilité au commerce des pelleteries, et qu'on ne distingue pas ses propres colons des sauvages" ? Va-t-on jusqu'à "supposer à un Canadien une figure extraordinaire et des mœurs encore plus étranges" ? ${ }^{1}$

${ }^{1}$ Pouchot, Mémoires sur la dernière Guerre de l'Amérique du Nord..., (Yverdon, 1781), I : xxxiv. 
Non, sans doute, quoique persistent, même chez les gens instruits, force préjugés, maintes erreurs, imputables dans une large mesure à un mànque de documentation sérieuse et bien à jour. De 1800 à 1830, en effet, peu de livres français s'attachent à décrire le Canada, et ceux qu'on rencontre sont pour la plupart des œuvres de seconde main, - traductions ou adaptations d'ouvrages anglais. Or, soit mépris de la race, soit ignorance de la langue, leurs auteurs se sont généralement peu souciés d'observer de près ces descendants des 60,000 Français restés au pays après la cession.

Les grandes Géographies de Mentelle et de Malte-Brun ${ }^{2}$ reflètent assez bien cet état d'esprit. A-peu-près, considérations vagues et superficielles y abondent: les Canadiens français "ne sont pas défricheurs"; "ils se contentent de retourner la terre avec la charrue". Ils n'ouvrent pas les fenêtres de leurs habitations, où l'on respire "un air fétide" . . . Dans leur jeunesse, "les villageoises françaises sont fort jolies". Les classes supérieures restent "obstinément attachées" à la langue française, etc.: tous lieux communs qui se trouvent déjà dans Weld, Heriot, Francis Hall, Talbot et quelques autres. Les éditions suivantes ne s'amenderont guère. Celle de $1854^{3}$ fera même l'objet d'une protestation de la Société historique de Montréal: "M. Danis signale cette Géographie qui nous donne pour des Métis."

D'Orbigny ${ }^{5}$ devait se montrer plus juste, après avoir, en 1836, traité bien à la légère "ces hommes rudes et sauvages qui parlent une espèce de jargon français" " ${ }^{6}$. L'édition de 1854, "augmentée de renseignements exacts jusqu'en 1853", s'inspire enfin des récits de voyageurs français contemporains. Les Canadiens, assure-t-on, "ne considèrent comme vraiment supé-

2 Mentelle et Malte-Brun, Géographie mathématique..., (Paris, Tardieu, an XII (1804). Cf. XIV: 194 sq.; 4e éd. (1836).

3 Op. cit., 6e éd. (1854).

4 Comptes-rendus (ms.), à la date du 29 déc. 1869.

5 Alcide Dessalines d'Orbigny (1802-1857) comptait de nombreux collaborateurs.

${ }^{6}$ Voyage pittoresque ..., in-4, 515 . 
rieur que ce qui vient de France". Chez les cultivateurs "le nom de Napoléon est vénéré, et beaucoup de jeunes gens le portent" (ce qui, pour l'auteur, était une manière comme une autre de faire sa cour !). Le fermier "qui n'aime pas aller à pied", s'attire même, en passant, ce petit couplet: "Quel air plein de fierté a le Canadien conduisant son cheval chétif et sa voiture mal assurée !" 7

Deux ouvrages de cette époque méritent cependant une mention à part: les Beautés de l'histoire du Canada, de D. Dainville, et le Tableau statistique et politique des deux Canadas, par Isidore Lebrun.

Ce Lebrun, aujourd'hui bien oublié, s'annonce sur la pagetitre comme "membre de plusieurs sociétés savantes". Plus tard, il devait accueillir favorablement, dans la Revue encyclopédique, les premiers tomes de l'Histoire du Canada, par Garneau ${ }^{8}$. Son Tableau, pour l'époque, est bien fait: rien de personnel, assurément, mais une honnête documentation tirée des auteurs déjà signalés et peut-être même, parfois, obtenue directement de source canadienne. L'édition est typographiquement belle. On regrettera d'autant plus la rhétorique, les digressions, les amphigouris qui déparent le style.

Qui était "D. Dainville", appellation qui a priori fleure le pseudonyme? Les érudits canadiens sont d'accord pour attribuer les Beautés à l'un des libraires Bossange, dont la maison jouissait, à Montréal comme à Paris, d'une grande vogue. L'ouvrage, édité chez eux et enrichi de gravures, est particulièrement soigné. D'après certains, l'auteur serait “Gustave Bossange, libraire à Paris" 9, mais le seul Gustave de la famille, agent à Paris, vers 1873, de l'immigration canadienne, vécut de 1837 à $1900^{10}$. Il ne saurait donc avoir écrit un livre paru en 1821 !

7 Voyage dans les deux Amériques..., (1854) in-4, 513.

8 Isidore Lebrun, Tableau statistique et politique des deux Canadas...

9 Audette et Malchelosse, Pseudonymes canadiens (Montréal, 1936), 45. 10 Communication de M. Jacques Lesourd, descendant des Bossange. 
Il faut plutôt voir là, croyons-nous, l'œuvre de son oncle Adolphe (1797-1862), qui signa quelquefois Nemo, collabora à la Gazette de France et fit jouer des pièces de théâtre avant d'entrer dans les affaires et devenir directeur des chemins de fer de l'Est. Il voyagea beaucoup dans sa jeunesse et visita à plus d'une reprise la succursale de Montréal. En cette même année, il devait publier et préfacer une édition de Volney.

Les Beautés, compilation assez terne dans l'ensemble, assument un ton plus vif, voire enthousiaste, dès qu'il s'agit des mœurs et de l'histoire des peaux-rouges, "mélange de ce qu'il y a de plus généreux et de plus atroce" "11. C'est d'ailleurs la partie la plus développée: deux cent cinquante pages sur un peu plus de cinq cents.

On ne trouve guère à signaler, vers cette époque, d'études sur l'histoire du pays, à moins que l'on n'admette comme œuvre historique le livre indigeste et plein d'erreurs de l'abbé Brasseur de Bourbourg, lequel s'attira de la part d'un confrère canadien, l'abbé Ferland, de l'université Laval, ce qu'on appellerait aujourd'hui un bel "éreintement" 12 .

Bien émouvante, au contraire, s'avère l'action d'un jeune professeur de Saint-Cyr, Louis-Étienne Dussieux. "En 1850, écrit-il, j'avais à exposer pour la première fois notre histoire nationale aux élèves de l'École... Lorsque j'en vins au récit de la lutte qui a coûté le Canada, l'ardente et sympathique jeunesse qui m'écoutait tressaillit. Je n'oublierai pas l'émotion qui s'empara de l'auditoire lorsque je dis que cette belle page de nos annales militaires était pourtant presque inconnue en France ${ }^{13}$." Dussieux réunit ses cours dans un petit volume paru en 1855, réédité et augmenté en $1862^{14}$.

11 Beautés du Canada (Paris, in-12, 1821), 110.

12 Brasseur de Bourbourg, Histoire du Canada et de son Eglise, de ses Missions ... (Paris), et Ferland, Observations sur un ouvrage . . ('Québec, 1853). $1855), 1$.

13 Dussieux, Le Canada sous la domination française (Paris, Tanera, 14 2e éd. (Lecoffre, Paris, 1862), 472 pp. 
Dans un autre genre, un Guide au Canada se trouvait dès 1829 à la disposition des touristes de langue française. Il s'intitulait Tournée à la mode ou Voyage de Charleston à Québec... C'était, naturellement, une traduction de l'anglais, mais l'auteur de cette version, un nommé Bourgeois, y avait mis une certaine élégance ${ }^{15}$.

Toutefois, l'image fidèle et vivante de cette NouvelleFrance, cédée à l'Angleterre mais jamais entièrement conquise, n'est-ce pas plutôt aux témoins oculaires qu'il faut la demander, aux voyageurs français qui se sont aventurés dans cette "Amérique britannique du Nord", comme on l'appelle officiellement?

Plusieurs bibliothèques de la Restauration possèdent déjà, sans doute, les relations de ces "exilés" plus ou moins volontaires de la Révolution, qui, à l'instar de René, le plus illustre d'entre eux, iront promener leur nostalgie sur les côtes américaines. Mais on ne peut guère se fier à leurs rapports en ce qui concerne les Français du Canada, car il est notoire que les Anglais ne laissaient pas encore les étrangers pénétrer jusqu'aux rives du Saint-Laurent.

Si tant est qu'il y posa le pied, en 1791, Chateaubriand luimême vit peu de chose de ces terres dont il déplorera pourtant la perte: "En regardant, dit-il, sur les vieilles cartes l'étendue des anciennes colonies françaises en Amérique, je me demandais comment le gouvernement de mon pays avait pu laisser périr ces colonies, qui seraient aujourd'hui pour nous une source inépuisable de prospérité2 $2^{6}$." Dans le Génie, il avait déjà consacré aux missions de la Nouvelle-France un chapitre où la fantaisie se mêle à la vérité historique, et où il célèbre l'héroîsme des jésuites qui "réchauffèrent de leur sang" ces "sillons glacés" ${ }^{17}$.

Volney ne visita certainement pas le Canada, mais il fut à même d'observer quelques Canadiens. Philippe Aubert de Gaspé,

15 Tournée à la mode dans les Etats-Unis ...

16 Mémoires d'Outre-Tombe (éd. Garnier), I: 393.

17 Génie du Christianisme (1re éd.), IV : 197. 
dans ses Mémoires ${ }^{18}$, nous dit que Mme Dupéron-Baby, grand'mère de sa femme, avait conservé un souvenir très précis de l'auteur des Ruines, qu'elle rencontra un jour d'automne de 1796, sur un bateau qui faisait le service du lac Érié. Le temps était mauvais, et Volney, caractère morose, malade de surcroît, se montra fort poltron. Dans son ouvrage sur les États-Unis ${ }^{19}$, où il ne veut indisposer personne, il vante la douceur, l'humeur sociable des Canadiens qu'il avait connus en Louisiane; mais, dans une lettre intime adressée de Philadelphie le 14 janvier 1797, il écrira perfidement: "J'ai vu dans le Canada les Français de Louis XV devenus demi-indien ${ }^{20}$."

On aimerait connaître les impressions personnelles du duc de la Rochefoucauld-Liancourt ${ }^{21}$, de passage sur le lac Ontario en juin 1795. Mais il a soin de nous avertir qu'il donnera sur toutes choses "l'opinion qui prévaut", n'ayant pas été autorisé à se rendre au-delà d'York (Toronto) et de Kingston. Bien reçu par Simcoe, lieutenant-gouverneur du Haut-Canada, il brûlait de pousser jusqu'à Montréal et Québec. Il en fit la demande à lord Dorchester, gouverneur général, mais essuya un refus. Comme le gentilhomme insistait, on lui répondit par un ordre d'expulsion! Aussi bien, lorsque La Rochefoucauld nous parle, par exemple, de l'ignorance "absolue" du clergé nous pouvons le croire mal renseigné, de même que s'il nous affirme qu'en 1790, année où il était encore en France, le thermomètre canadien "a descendu au-dessous de toute graduation, et le mercure est rentré dans la boule" ${ }^{22}$ !

M. Guénard-Hodent, qui nous conta jadis les tribulations du malheureux duc, croyait qu'un sien compagnon de voyage, Français comme lui, avait été plus heureux en forçant les con-

18 Cité par M. Trudel, L'influence de Voltaire au Canada (Montréal, 1945), I: 43.

19 Tableau du climat et du sol des Etats-Unis ... Cf. II : 415.

20 Dans G. Chinard, Volney et l'Amérique (Baltimore, 1923), 64.

21 François, duc de LaRochefoucauld-Liancourt (1747-1827), voyageur et philanthrope. Son Voyage dans les Etats-Unis ... parut à Paris en 1798. 22 Voyage, II : 207. 
signes; mais ce Guillemard, malgré son nom, était Anglais et descendant de huguenots ${ }^{23}$.

Il semble donc que le premier notable français à revoir le Bas-Canada après la cession ait été, en 1798, Édouard-CharlesVicturnien Colbert, comte de Maulevrier ${ }^{24}$. C'est M. Gilbert Chinard qui, en 1935, nous a restitué les "petits carnets" où le noble exilé, "voyageant seul, en touriste et en artiste plus qu'en observateur politique", note ses impressions "au jour le jour, avec la minutie et l'exactitude d'un marin qui tient son journal de bord". On comprend que ce gentilhomme, sociable et "sensible" comme l'étaient ceux de son siècle, n'ait pu visiter "sans regret et sans tristesse" l'ancienne colonie de Louis XV.

Aussi ses observations valent-elles qu'on s'y arrête, quoique le personnage n'appartienne pas encore à l'époque qui doit nous occuper particulièrement. Arrivé à Montréal par la voie des Grands Lacs, le 31 octobre 1798, en partira pour Québec le 10 novembre, nanti d'une permission toute spéciale du gouverneur Prescott, car Québec, ville fortifiée et séjour de la garnison, est bien gardée. Le voyage est assez pénible, sur un méchant sloop "dont les voiles et les cordages sont pourris". On a du gros temps sur le lac Saint-Pierre, et le Français, "en vrai matelot", doit aider à la manœuvre. Il y laisse son chapeau "que le vent a emporté !" Le froid se fait sentir et les rues escarpées de Québec "ne sont qu'une glace”. Pour s'y aventurer, il faut s'armer les pieds de fers, "comme les chevaux !" ${ }^{25}$

Le comte de Maulevrier n'est pas sans préjugés. Comme son prédécesseur La Rochefoucauld (endoctriné par les Anglais), il croit le clergé canadien "très ignorant", mais il n'a certainement pas rencontré l'évêque ni les prêtres de l'un ou l'autre

23 Guénard [-Hodent], "Le tourisme au Canada il y a cent ans", Revue France-Amérique (août 1926), 266.

24 Colbert de Maulevrier (1758-1820) entra dans la Marine royale, prit part à la guerre d'Amérique, passa aux E.-U. en 1796, rentra en France en 1799, se tint à l'écart jusqu'en 1815, alors qu'il devint député d'Eure-et-Loir, puis commandeur de Saint-Louis et contre-amiral.

25 Voyage dans l'intérieur des Etats-Unis et du Canada... (Baltimore, 1935). Introd., xviii, puis, 63-69. 
Séminaire. Parlant de "quelques ecclésiastiques que la Révolution a jetés au Canada", ne va-t-il pas jusqu'à affirmer que, n'était la présence au pays de ces nouveaux venus, "il n'y aurait pas dix sermons de prêchés par an dans tout le Bas-Canada" !

Écoutons plutôt Colbert nous entretenir de ce qui est de sa compétence. Personne, ici, assure-t-il, ne veut ajouter foi à la mort de Louis XVI. Malgré les témoignages du clergé et des gouvernants anglais, on ne peut croire le peuple français "capable des horreurs qu'il a commises". Ce qui, d'après notre auteur, démontre suffisamment que "le Canadien est encore attaché à la France, et non à l'Angleterre".

Il pense que, lors de l'expédition de Richery à Terre-Neuve, en 1795, les Républicains français n'auraient eu qu'à débarquer 6.000 hommes au Canada pour reprendre la colonie à l'Angleterre. Mais "c'eût été un malheur pour ce pays", car c'est peutêtre le seul "où l'on n'ait rien souffert des convulsions qui agitent [l'Europe] depuis dix ans". D'autre part, le Canada eût été "perdu", en passant aux Etats-Unis: alors que "les taxes commencent à peser sur le peuple américain", lui "ne paie aucun impôt et jouit de la protection d'un gouvernement solide et considéré".

Enfin, Colbert de Maulevrier, en quittant le pays par SaintJean, le 8 décembre 1798, lui rendit cet hommage appréciable: "Je puis dire [y] avoir reçu plus d'honnêtetés et de marques de bienveillance dans l'espace de deux mois environ, que je n'en ai éprouvées depuis près de trois ans en Amérique ${ }^{26}$."

Ainsi se clôt abruptement, pour trente ans à venir, la série de nos témoins. Les rapports entre l'Angleterre et la France républicaine se font de plus en plus mauvais: la première bataille d'Aboukir est du mois d'août 1798. Ensuite, sous le Consulat et l'Empire, Napoléon devait laisser à ses sujets bien peu de loisirs pour les voyages d'agrément! Même après Waterloo et le Congrès de Vienne, les Français qui, à l'exemple du baron de

26 Op. cit., 69. 
Montlezun ${ }^{27}$, entreprendront de lointaines randonnées aux ÉtatsUnis et en d'autres contrées d'Amérique, ne paraîtront pas très pressés de revoir l'ancienne colonie. Il se peut bien, toutefois, que des notes inédites et insoupçonnées, égarées dans des papiers de famille, nous soient révélées quelque jour.

Mais, pour retrouver un ouvrage dûment composé et imprimé, il faut attendre jusqu'aux Souvenirs atlantiques de Théodore Pavie ${ }^{28}$. Quand le jeune Angevin visita les régions de Montréal et de Québec, en juin 1829, il y avait soixante-dix ans que Montcalm avait perdu la bataille des Plaines d'Abraham et que le chevalier de Lévis, plutôt que de rendre au vainqueur les drapeaux français, était allé les brûler ou les enfouir en quelque lieu secret.

Qu'étaient devenues, depuis le traité de Paris, ces populations abandonnées ? Les Canadiens avaient-ils été absorbés par le conquérant, ou restaient-ils, au contraire, fidèles aux souvenirs français, et dans quelle mesure ? C'est alors seulement que la question devient intéressante. Avant 1760, on ne se souciait guère de la poser, pas plus qu'aujourd'hui on ne se demande jusqu'à quel point les blancs de la Martinique ou de la Guadeloupe sont demeurés français. Tandis que le cas de la petite île Maurice et celui du vaste Canada, englobés tous deux dans l'Empire britannique, ont fait et font toujours la surprise et l'admiration de la Métropole.

Le premier Français à constater au Canada cette survivance et à la célébrer, fut donc Théodore Pavie . . . "Écolier de la veille", comme il s'intitule lui-même, il était né à Angers, dixhuit ans plus tôt, d'une famille qui jouissait à la fois d'une large aisance et de l'estime générale. Les Pavie, comme on sait, furent en relations suivies avec maints romantiques, dont Victor Hugo, les Foucher, Sainte-Beuve et le sculpteur David. Leur compa-

27 Baron de Montlezun, Voyage fait, les années 1816 et 1817, de NewYork à la Nouvelle-Orléans... (Paris, 1819). On pourrait mentionner aussi E. de Montulé, qui ne vit le Canada que depuis les chutes du Niagara.

28 Théodore Pavie, Souvenirs atlantiques... (Bossange, Paris, 1833). 
triote René Bazin nous a laissé sur eux, et notamment sur Thédore, des souvenirs intéressants ${ }^{29}$.

Ce voyage aux États-Unis et au Canada fut pour le jeune Pavie une aventure peu banale. Assurément, tous les Français de son âge ne pouvaient, aussitôt terminées leurs études secondaires, se payer pareil luxe! Et puis combien eussent pu s'enorgueillir de posséder un cousin planteur à la Rivière-Rouge, et, au retour, un père tout ensemble imprimeur, "lettré, botaniste et même un peu poète" ${ }^{30}$ ?

Remarquons en passant que ce titre de Souvenirs atlantiques, qui implique une idée de rétrospection, n'est pas très juste, car ces observations, jetées sur des carnets d'avril 1829 à juiliet 1830 et revisées en rentrant, parurent dès 1832 à Angers en un premier tirage intime. L'année suivante, Hector Bossange devait se charger de l'édition parisienne. Paysages ou Visions eût donc mieux exprimé l'actualité de ces impressions ${ }^{31}$.

C'est bien cela, d'ailleurs, que Théodore compte nous offrir: "Je ne ferai, avoue-t-il, que reproduire naïvement ce que j'ai vu et senti." A-t-il bien vu et bien senti ? Il serait excessif de dire qu'il est allé au fond des choses. Ses appréciations sont superficielles, sa topographie manque de précision: il emploie à tort et à travers des noms de lieux parce qu'ils sont sonores, il croit apercevoir "la côte d'Acadie", alors qu'il s'agit d'un simple village appelé L'Acadie, etc. Ce qui, évidemment, le charme par-dessus tout, c'est le cadre naturel du pays, l'hospitalité et les mœurs patriarcales de ces anciens Français, et d'abord les côtés poétiques de leur existence. Romantique, il l'est irrésistiblement. Il citera Byron, Lamartine. Son premier soin, en débarquant à Bordeaux, sera d'aller applaudir Hernani au Grand Théâtre !

Le style se ressent des tendances de l'époque et de la jeunesse de l'auteur: il est d'un rhétoricien, mais fort en thème,

\footnotetext{
29 Bazin, "Théodore Pavie, dans "Cent ans de vie française" à la Revue des Deux Mondes (Paris, 1929), 102-111.

30 Bazin, op. cit., 105.

31 Le titre choisi, paraît-il, aurait été suggéré par David d'Angers (A. Crosnier, Théodore Pavie, Angers, 1897).
} 
prodigue de comparaisons et désireux de "faire pittoresque" comme de s'émerveiller lui-même. A bord, les vagues étaient déjà "ces cohortes de flots pressés"; plus tard, le clocher de Varennes lui apparaîtra de loin comme "l'aigrette d'un chef algonquin" !

A Montréal, le 7 juin 1829, il devait assister à une "pompeuse cérémonie": la bénédiction solennelle de l'église NotreDame, paroisse sulpicienne, qui était alors et allait demeurer longtemps le plus vaste temple de l'Amérique. La présence du jeune Français à cette fête religieuse est un détail curieux que les Canadiens aimeront enregistrer ... À Québec, ville "majestueuse", ceinte de ses murailles "crénelées" (sic) "comme d'un turban" il constate après Maulevrier que les militaires sont sur le qui-vive: on y a toujours, dit-il, "une extrême défiance des étrangers, et surtout des Français". Il se moque en passant des "jeunes officiers anglais, dans toute l'exagération de leur costume ..., raides comme des thuyas du Saint-Laurent et serrés comme des maringouins" !

Ce qu'il y a de plus sérieux, et même de touchant, chez Théodore Pavie, c'est le souvenir sympathique qu'il garda toujours aux Canadiens français. Devenu un orientaliste distingué, il évoquera à trois reprises au moins, en 1850, 1853 et 1865, dans la Revue des Deux Mondes, le pays et les habitants qu'il avait admirés dans sa prime jeunesse ${ }^{32}$. Ce chrétien convaincu, cet homme simple et serviable, mais vif et spontané, dont "les yeux d'un bleu de mer pétillaient d'esprit" ${ }^{33}$, s'éteignit en 1896, à l'âge de 85 ans, dans son domaine de la Chaufournaie ${ }^{34}$.

Deux ans plus tard, en août 1831, le Canada recevait la visite d'Alexis de Tocqueville. Accompagné de son ami Gustave de Beaumont, le jeune économiste, quelques mois auparavant, avait abordé aux États-Unis dans le but avoué d'y étudier le régime pénitentiaire; mais cet objectif devait être largement

32 R.D.M., $\mathrm{n}^{\text {os }}$ des $1^{\mathrm{er}}$ août et 15 déc. 1850, 15 juil. 1853, 15 janv. 1865.

33 Bazin, op. cit., 104.

34 Souvenirs atlantiques... Nos citations: I : 174, 13, 159, 189, 145. 
dépassé, quand, à son ouvrage sur cette question subsidiaire, succéda La Démocratie en Amérique.

On sait que, par la suite, Tocqueville jugera avec sévérité le régime imposé jadis par la France à son ancienne colonie. Mais, pour connaître ses impressions directes du Canada, il faut puiser dans l'édition monumentale et définitive de ses Euvres complètes, en cours de publication sous la direction de M. J.-P. Mayer ${ }^{35}$... Ses procédés d'enquête nous étonnent, car ils sont déjà ceux de nos reporters d'aujourd'hui. Ainsi, il se ménagera de véritables interviews avec les personnages les plus divers, leur posant des questions pertinentes et précises. Nous connaissons, entre autres, les réponses que lui firent des sauvages, un bois-brûlé, un négociant anglais, les deux frères Mondelet, futurs juges, le député John Neilson, d'origine écossaise, enfin le Supérieur du Séminaire de Montréal, M. Quiblier, "ecclésiastique aimable et éclairé" ${ }^{36}$.

Grand liseur, déjà pourvu d'une forte culture générale, notre voyageur pourra, en une quinzaine bien remplie, se rendre suffisamment compte de ce qu'était le Canada six ans avant la rébellion de 1837. L'état d'infériorité imposé aux Canadiens français par les Anglais saute aux yeux. "Je ne puis croire, assure-t-il, que [les deux peuples] se fondent jamais, ni qu'il puisse exister une union indissoluble entre eux"... Il espère même que "les Français, en dépit de la conquête, arriveront un jour à former à eux seuls un bel Empire dans le Nouveau Monde" ${ }^{37}$ ! Mais, n'ayant encore vu dans le Canada "aucun homme de talent, ni lu une production qui en fît preuve", il conclut: "Celui qui doit remuer la population française et la lever contre les Anglais n'est pas encore né ${ }^{38}$." Évidemment, il n'avait pas eu l'heur de rencontrer Louis-Joseph Papineau !

Des lectures imprudentes avaient fait perdre au jeune Alexis la pratique religieuse sinon la foi. Néanmoins, il rendra justice

${ }^{35}$ Gallimard éd. Nos citations sont tirées principalement du $t$. V (Voyages, Sicile et Etats-Unis).

36 Voyages, $1^{\text {re }}$ partie, 77 .

37 Op. cit., 210.

38 Op. cit., 212. 
à la piété des Canadiens, qui lui a paru "éclairée" et de bon aloi. Là, pas d'"industrie religieuse" comme chez les protestants du pays voisin. "Le curé est bien véritablement ici le pasteur du troupeau ... Ou il faut nier l'utilité du clergé, ou il faut l'avoir comme au Canada ${ }^{39}$."

Tocqueville, quand il comparera les deux pays, donnera souvent la préférence au Canada. Témoin ce passage: "Les villages que nous avons vus aux environs [de Québec] ressemblent extraordinairement à nos plus beaux villages. On n'y parle que le français. La population paraît heureuse et aisée. Le sang y est remarquablement plus beau qu'aux États-Unis. La race y est forte, les femmes n'ont pas cet air délicat et maladif qui caractérise la plupart des Américaines ${ }^{40}$."

Sans doute connaîtrons-nous encore mieux la pensée entière d'Alexis de Tocqueville, quand aura paru dans son intégrité la Correspondance, et surtout les lettres qu'il adressait fréquemment à son ancien précepteur, l'abbé Lesueur.

Quoi qu'il en soit, avec son talent de bien regarder et de rechercher le pourquoi des choses, un bref séjour lui a suffi pour reconnaître dans le Bas-Canada de véritable frères par le sang et par la pensée: “On ne peut contester leur origine, écrira-t-il à un intime, ils sont aussi Français que vous et moi ${ }^{41}$." Et encore, à son précepteur: "Nous nous sentons chez nous, et partout on nous reçoit en compatriotes, en fils de la vieille France. À mon avis, cette épithète est mal choisie: la vieille France, elle est au Canada, et la nouvelle est chez nous ${ }^{42}$."

Un autre économiste de même âge mais d'opinions bien différentes, le saint-simonien Michel Chevalier ${ }^{43}$ passa, en 1833 et

39 Op. cit., 211.

$40 \mathrm{Op}$. cit., 211. Quand G. de Beaumont entreprit (vers 1865) l'édition des œuvres ms. de son ami, il omit ces deux dernières phrases, de peur, sans doute, de désobliger leurs anciens amis des Etats-Unis!

41 Lettre inédite, communiquée par M. Henri Chardon au journal le Canada ( $\mathrm{n}^{\circ}$ du 18 juin 1935$)$.

42 Citée par G. W. Pierson, Tocqueville \& Beaumont in America (N.Y., Oxford Press, 1938).

43 M. Chevalier, Lettres sur l'Amérique du Nord... (1936). 
1834, près de deux ans aux États-Unis, pour y observer de près les chemins de fer. Les lettres qu'il adressa au Journal des Débats furent remarquées et les critiques américains eux-mêmes y reconnurent un tableau fidèle de la vie sociale d'alors ${ }^{44}$. L'auteur ne se fait pas faute, à l'occasion, d'y décrocher quelques traits acérés aux Canadiens français qu'il n'a peut-être pas vus chez eux. Leur infériorité par rapport aux Anglo-Saxons lui apparaît surtout dans leur habitude de faire travailler les femmes! Il les considère d'ailleurs comme voués à une anglicisation totale ${ }^{45}$.

Quand nous aurons à parler de la rébellion de 1837-1838, il sera utile de citer les observations de Charles-Édouard de Pontois, ambassadeur de France à Washington. Le diplomate ne fut pas à vrai dire témoin des "troubles" qui n'éclatèrent qu'en novembre, car c'est en juillet 1837 que, en compagnie de son secrétaire Dubois de Saligny, il avait passé deux semaines à Montréal et à Québec. Mais la tension politique durait au moins depuis 1834, et il était déjà facile, surtout pour un homme du métier, de prévoir une catastrophe prochaine. De Pontois n'eut garde d'y manquer. Comme l'ambassadeur arrivait chaudement recommandé par Fox à lord Gosford, on peut deviner en quel sens il écrira à son ministre, le comte Molé ${ }^{46}$.

Une fois vaincus et condamnés, les rebelles, nous le verrons, éveilleront la sympathie de maints Français de France, d'Alfred de Vigny, entre autres, et l'un d'eux ira intercéder jusqu'en Angleterre en faveur des déportés: c'était lui-même un exilé volontaire, Mgr de Forbin-Janson.

Mais c'est à un tout autre titre qu'il importe de mentionner ici le voyage de l'évêque de Nancy et Toul. Le 18 octobre 1839, Mgr de Forbin-Janson débarquait à New-York, autorisé par le Pape à entreprendre une longue tournée missionnaire en Amé-

44 Dans ses French Travellers in the U.S., M. F. Monaghan ne craint pas d'affirmer: "[Chevalier] remains one of the most important commentators on the United States" (p. 25).

45 Chevalier, op. cit., (éd. Gosselin, 1836), II : 111, 226-227.

${ }^{46}$ Les Lettres de Pontois à son Ministre ont été publiées par M. de Roquebrune dans Nova Francia (1927), III: 238 sq. 
rique du Nord. Le 3 septembre 1840, il est au Canada, où il demeurera jusqu'au 5 novembre de l'année suivante, ne s'absentant que pour des séjours momentanés aux États-Unis.

On sait que les opinions ouvertement légitimistes du prélat lui rendaient de plus en plus difficile, depuis 1830, la résidence à Nancy. Faute de souplesse et de doigté, il s'était aliéné une notable partie de son clergé ${ }^{47}$. Peu instruit de ces détails, le peuple canadien n'ignorait pas que des ennemis de l'Église avaient contribué à brouiller les cartes, et cela, à ses yeux, suffisait à conférer à l'évêque quelque chose de l'auréole du martyre. Et puis, le Canada allait connaître surtout son zèle apostolique, et celui-ci était sans bornes.

Cette "mission" mémorable, dont le souvenir persistant n'est pas tout à fait effacé chez les fils et les petits-enfants de ceux qui y participèrent, prit une envergure immense et remua profondément les masses. Se dépensant au point de compromettre à jamais sa santé, le prédicateur est partout, - dans les villes pour y parler aux prêtres comme aux fidèles, fonder des ligues de tempérance et installer même un chapitre; dans de nombreux villages tels que Châteauguay, Vaudreuil, Rigaud, Saint-Benoît, Belœil, où, en plein air, des foules qu'il estime (non sans exagération) à dix, douze et même trente mille auditeurs, se pressent à ses pieds et rappellent les plus beaux jours des temps évangéliques. Ce qui fera s'écrier au père Lacordaire, prononçant en 1844 l'éloge funèbre de l'évêque, que celui-ci avait choisi "le Canada, qui est une terre française, pour le théâtre principal de ses courses apostoliques", et que là, "le sommet des montagnes, le bord des fleuves et des lacs lui servaient de basiliques, à défaut des églises devenues trop étroites" ${ }^{48}$.

Accueilli à bras ouverts par l'épiscopat et tout le clergé, entouré du respect d'une population qui l'écoutait avec tant d'a-

47 Sur Mgr de Forbin-Janson en général, cf. P. Lesourd, Un grand cœur missionnaire (Paris, s.d. [1944]), et sur l'œuvre accomplie au Canada : F.-X. Côté, "Mgr de F.-J. et le mouvement religieux du Québec vers 1840" (Soc. can. d'hist. de l'Eglise (1941-42), 99-114).

48 Lacordaire, Notices et panégyriques (Paris, Poussielgue, 1920), 106. 
vidité, Mgr de Forbin-Janson devait réciproquement goûter, avec la foi vive et la belle docilité des Canadiens, leur attachement à tout ce qui évoquait la France d'ancien régime. "Peuple que j'aime et dont je suis certain d'être aimé", dira-t-il de "ses Canadiens aux cœurs d'or et aux clochers d'argent 49." À la veille même de sa fin, son entourage remarquera qu'il n'a "que deux sujets de conversation: les Canadiens et la Sainte-Enfance" ${ }^{50}$.

Publiés en 1842, les Vues et Souvenirs de l'Amérique du Nord, par le comte Francis de Castelnau ${ }^{51}$, doivent remonter à 1838, puisque l'auteur assista à de bruyantes démonstrations en faveur du gouverneur lord Durham. Ce fils naturel de George IV peint en traits justes, parfois finement observés, les Canadiens français, qu'il a beaucoup mieux compris que Pontois, son collègue dans la carrière diplomatique.

Il paraîtra plus difficile de dater exactement le passage au Canada d'un nommé Étourneau, qui prétend nous offrir "la narration d'un voyage de dix ans", commencé en 1837. Se paret-il d'un pseudonyme ? en ce cas, il l'aurait admirablement choisi, car son texte ne brille ni par le sérieux ni par l'objectivité. Il avait d'abord lancé, en 1855, un simple Livret-guide de l'émigrant, où, pour mieux exalter les vertus anglo-saxonnes, il rabaissait les Canadiens français au rang d'une "race primitive" 52. Quelques années plus tard, il se ravisa et, s'inspirant sans doute des notes de ses prédécesseurs plus que des siennes propres, il donna De Paris au Nouveau Monde et du Nouveau Monde à Paris, en trois volumes ${ }^{53}$.

A ce prolixe ouvrage on préférera certainement les Lettres sur l'Amérique, de l'honnête et bienveillant Xavier Marmier ${ }^{54}$.

${ }^{49}$ Lettre citée par N.-E. Dionne, Mgr de Forbin-Janson (Québec, 1910), 146.

50 Lesourd, op. cit., 145.

51 Francis-Louis-Nompar de Caumont-Laforce, comte de Castelnau, né à Londres en 1812; mort consul de France à Melbourne, en 1880.

52 Livret-guide ..., 67.

53 De Paris au Nouveau... (Paris, 1857-59).

54 Xavier Marmier, Lettres sur l'Amérique (Paris, s.d. [1851]), 2 v. 
Cet académicien, tombé depuis sa mort, et même auparavant, dans un oubli complet, jouit en son temps d'une certaine notoriété. On lui rendra cet hommage d'avoir été "l'un des pionniers de la littérature comparée" ${ }^{55}$, encore que ses traductions des folklores étrangers nous paraissent aujourd'hui manquer de nerf et de couleur.

Royaliste et catholique militant, Marmier était tout disposé à voir les beaux côtés du Canada français. Mal impressionné par la Révolution de février 1848, il s'embarqua l'année suivante sur un paquebot qui le conduisit à New-York. Il ne tarda pas à passer au Canada, d'où il adressa à une certaine "Madame E. R. de F." plusieurs de ses lettres parues en $1851 \mathrm{chez}$ Bertrand. Il ne visita pas seulement Montréal et Québec, comme tant de voyageurs pressés, mais encore des villes de moindre importance, telles que Saint-Hyacinthe, et s'attarda volontiers dans les campagnes, qu'il décrit à loisir, truffant ses observations d'anecdotes, de légendes et même de réminiscences classiques.

Son public était surtout bourgeois. Un critique américain, M. Frank Monaghan, pense avec raison que cet écrivain fut de ceux qui firent le mieux connaître l'Amérique en France, vers cette époque ${ }^{56}$. Le bon Marmier, qui avait voyagé pendant quarante ans, passa le reste de sa vie à refondre, à remanier ses vieux ouvrages auxquels il donna de nouveaux titres. Il eut ainsi l'art de faire double et même triple mouture, sans profit aucun pour le lecteur. Il était, à l'Académie française, le dévoué serviteur des intérêts canadiens-français.

Devenu conservateur, puis administrateur de la bibliothèque Sainte-Geneviève, il put ainsi satisfaire son amour des livres. On se souvient peut-être que, bouquineur enragé et ami des bouquinistes, il légua à ceux-ci, par testament, "mille francs pour faire un bon dîner" ${ }^{57}$ ! 167.

55 C. Aymonier, Xavier Marmier, sa vie, son œuvre (Besançon, 1928),

56 "His books concerning America were not without a marked influence upon French opinion" (French Travellers... (New York, 66).

57 Aymonier, op. cit., 167. 
On peut attribuer à Marmier, croyons-nous, une plaquette in-octavo de 36 pages, publiée à Paris en 1854: Le Canada, ou quelques mots de réfutation à $M$. le pasteur Grandpierre, par "Un ami des Canadiens français". Un peu auparavant, un ministre français du culte réformé était allé visiter diverses communautés protestantes des Etats-Unis. Ses impressions avaient paru dans le journal L'Espérance, puis en volume sous le titre de Quelques mois de séjour aux États-Unis d'Amérique. Rien à redire à cela, sinon que, pour un homme d'Église, l'auteur célébrait bien haut le confort et le luxe dont jouissaient les Américains. Toutefois, il avait ajouté une manière d'appendice sur les Canadiens français, qu'il semblait avoir peu hantés, s'il n'avait pas simplement enregistré sur leur compte des opinions toutes faites. Or, ces jugements étaient autant d'outrages, empreints du parti-pris le plus évident. Le pasteur, qui prétendait avoir trouvé ce peuple bien dégénéré et esclave de la routine (ce qu'il expliquait par "l'influence peu civilisatrice d'un clergé qui ne brille pas plus par ses lumières que par son zèle"), n'allait-il pas jusqu'à souhaiter que les Canadiens français "ne se rattachassent (sic) par aucun lien à ce pays de leurs pères, auquel ils sont loin de faire honneur" ${ }^{58}$ ? Puis, dans une conclusion qui dépassait évidemment les prémisses, notre révérend entonnait le refrain si souvent repris de la supériorité des peuples protestants sur les catholiques.

Les Canadiens (qui allaient pourtant en voir et en entendre bien d'autres, entre 1880 et la première grande Guerre !) furent profondément blessés. Or, le champion français qui a pris leur défense réfute d'abord, comme peu pertinents, des exemples tirés par le pasteur de la condition sociale de catholiques et protestants dans les divers cantons suisses. En faveur du peuple canadien, il cite ensuite de nombreux témoignages, dus à des autorités anglaises, américaines et françaises, - dont les appréciations de Marmier lui-même. Comme le style est identique au sien, que le ton assuré de l'ouvrage est celui de quelqu'un qui a vu de ses yeux et que, par ailleurs, Marmier était rentré depuis deux

${ }^{58}$ Quelques mois de séjour... (Paris, 1854), 131-134. 
ans de ses pérégrinations, nous pensons qu'il voulut bien rompre ces lances pour obliger ses amis d'outre-mer. Ajoutons que la brochure est ornée d'une seule gravure, et que celle-ci représente la petite ville de Saint-Hyacinthe, qu'avait grandement admirée notre voyageur.

Ce que Marmier venait de faire pour le cercle bourgeois et dévot de ses lecteurs, en leur révélant le Canada français, JeanJacques Ampère va le réaliser à son tour, en 1853, quand il offrira au public intellectuel et libéral de la Revue des Deux Mondes la primeur de sa Promenade en Amérique, bien des fois rééditée depuis ${ }^{59}$.

Curieuse figure que ce fils sentimental et littéraire d'un grand savant! L'Europe ne l'appelait que "le charmant Ampère". "Nature à la fois si riche et si éparse", dira de lui SainteBeuve, qui lui reprochera d" "avoir passé sa vie à se chercher" ${ }^{60}$. On pourrait ajouter: sans s'être jamais trouvé, puisque, malgré les "arriérés de tendresse" dont son âme était pleine, il mourut célibataire, après avoir vu disparaître les deux femmes qu'il aime d'un amour idéal.

En 1849, c'est Mme Récamier, "sa grande flamme" ${ }^{61}$, qu'il vient de perdre. Désemparé, il écrit dans son journal intime: "Ma vie est déracinée" 62 et se met à voyager pour s'étourdir, sur le continent d'abord, puis, en 1851, en Angleterre. De là, il s'embarque subitement pour l'Amérique, à l'insu même de ses meilleurs amis, Ozanam et Tocqueville.

Mais ce dernier, à qui il dédiera plus tard ses impressions de voyage, ne tardera pas à recevoir de ses nouvelles. Et Tocqueville, esprit toujours en éveil, en constante gestation, lui écrira à son tour, le 6 octobre: "Que je serai curieux, au retour, de vous faire causer sur les pays que vous êtes occupé à parcourir, de savoir ce que vous avez vu de même ou autrement que moi, ce

59 J.-J. Ampère, Promenade en Amérique, 2 v.

60 Etude, en tête de l'éd. in-4 de 1874, p. iv.

61 De Launay, Un amoureux de Mme Récamier: le Journal de J.-J. Ampère ... (Paris, Champion, 1927).

62 Op. cit., 286. 
qui est changé depuis vingt ans, non seulement dans l'aspect superficiel et matériel, mais aussi dans le fond; car il est impossible que le tempérament même des peuples ne s'altère pas au milieu d'une si rapide croissance ${ }^{63}$."

Jean-Jacques arriva dans le Bas-Canada à la fin de septembre 1851 , et y passa environ deux semaines. Ses premiers contacts avec des Canadiens remontaient déjà à quelques jours, car, à Boston, il avait pris part aux fêtes qui marquèrent l'inauguration de la voie ferrée reliant cette ville à la rive sud du Saint-Laurent. Il avait goûté l'entrain des Canadiens français, notant combien les Américains les tenaient en estime. Le banquet lui sembla particulièrement gai: n'y avait-on pas porté des toasts "à la belle France" et chanté de vieux refrains tels que A la claire fontaine et Vive la Canadienne?

Ampère fut accueilli avec déférence par l'élite canadiennefrançaise, qui saluait en lui le premier académicien à visiter le Canada. Il avait une lettre d'introduction, dit-il spirituellement, "pour M. Lafontaine, qui n'écrit pas des fables, mais qui est le chef d'un ministère libéral et modéré". Louis-Hippolyte Lafontaine (qui n'avait pas encore été créé baronnet), allait bientôt quitter l'arène politique. Il rencontra à maintes reprises son hôte de France, alla déambuler avec lui sur le versant du mont Royal, et, à son dîner d'adieu, le $1^{\text {er }}$ octobre, Ampère fut "placé à côté du héros de cette fête patriotique" ${ }^{64}$. Il put ainsi s'entretenir avec Georges-Étienne Cartier, Augustin-Norbert Morin et plusieurs autres personnalités du moment.

Cette "promenade", qu'Ampère a recontée "avec la même rapidité qu'il mit à la faire" ${ }^{65}$, se lit toujours avec agrément. Elle ne manque pas de pénétration ni de sel ${ }^{66}$. Sa verve légèrement caustique apparente Jean-Jacques au Taine des Notes

63 [Mme Cheuvreux], Souvenirs et Correspondance d'A.-M. et J.-J. Ampère (Paris Hetzel, 1875), II : 210.

64 Promenade ..., I: 107, 138.

65 Ed. 1874, Introd., xxxii.

66 "His observations are intelligent, sometimes shrewd, and often witty", dira également M. F. Monaghan (French Travellers ..., 4). 
sur l'Angleterre et nous laisse pressentir ce qu'eût écrit sur ce monde nouveau un Thomas Graindorge.

Les Canadiens français purent se flatter d'avoir été bien compris de ce fin littérateur. Tout en rendant hommage à leur amour des traditions ancestrales, il tâche de s'expliquer leur loyalisme envers la Couronne britannique, mais ne se dissimule pas non plus les graves difficultés qu'ils rencontrent dans le domaine politique. En outre, comme nous aurons bientôt l'occasion de le voir, il plaide chaleureusement pour de plus étroites relations officielles avec la France.

Jean-Jacques Ampère, disciple autant qu'ami d'Alexis de Tocqueville, pourra affirmer, dans sa dédicace à son maître ès sciences sociales: "Chaque jour, chaque heure passés aux ÉtatsUnis [et au Canada, sans doute], étaient un commentaire de voter œuvre, une vérification de vos pensées ${ }^{67}$." Mais, "avec ce don merveilleux de s'intéresser à tout" ${ }^{68}$ qui le caractérise, JeanJacques ne peut négliger le facteur religieux. Les origines foncièrement chrétiennes $d u$ peuple canadien ne lui ont point échappé. À son autre mentor, Frédéric Ozanam, qui se dira "charmé de le voir assis au banquet de famille de nos frères d'outre-mer" ${ }^{69}$, il écrit de Montréal, le 2 octobre 1851: "C'est l'Église qui a fait en grande partie ce pays. Elle y a joué, à peu de choses près, le rôle que vous avez si bien peint dans votre histoire des premiers siècles" ${ }^{70}$.

67 Promenade... (éd. 1856), I: v.

68 Ozanam, Lettres (Paris, de Gigord, 8e éd. 1912), II : 364.

69 Op. cit., II: 360 : lettre à Ampère, datée de Sceaux le 22 oct. 1851. En répondant à cette lettre de Québec, le voyageur affirmera à son ami que le nom d'Ozanam "est en grand honneur" au Canada (Cf. Cheuvreux, Souvenirs ..., II : 356 ).

70 Cheuvreux, op. cit., II : 355. Déjà citée par G. Goyau, dans Les Origines religieuses... (Paris, 1924), préf., xx, cette lettre débute ainsi: "C'est du Canada que je voulais dater la première lettre que je vous écrirais d'Amérique."

A cette liste de témoins, on pourrait ajouter: Adolphe de Puibusque (1801-1863), gentilhomme de belles manières et fervent catholique, critique littéraire estimé en son temps. Il plut infiniment pendant les deux années (entre 1850 et 1854) qu'il passa au Canada avec sa femme. En 1861 et 1862 , il publia dans les Causeries des Familles d'aimables tableautins de la vie canadienne. Après sa mort, sa bibliothèque (Catalogue de 2744 ouvrages) fut vendue. Elle contenait de nombreux "canadiana", dont certains reliés en peau de marsouin blanc du Saint-Laurent! 
Ainsi s'achève, avant 1855, un demi-siècle de "pénétration lente", au cours duquel l'opinion française, surtout au début, s'est trouvée bien peu renseignée sur les choses et les gens du Canada. Au poète Théodore Pavie, à Tocqueville, déjà économiste averti, et à l'aimable dilettante Jean-Jacques Ampère, vont succéder désormais de nombreux voyageurs qui brosseront $d u$ pays et de ses habitants un tableau sensiblement plus élaboré. Mais ce sera la continuation d'une "ère de sentiment", où nos témoins insisteront particulièrement sur la fidélité des Canadiens français aux traditions de la mère patrie.

C'est seulement vers 1880 , pour des motifs que nous exposerons, qu'on verra s'instaurer une période nouvelle, où le peuple canadien sera passé au crible d'une critique fréquente, souvent injuste, parfois salutaire.

Puis surviendra la première Guerre mondiale. Après une nouvelle "poussée" sentimentale, occasionnée par la participation des Canadiens et la révélation de Maria Chapdelaine, le Canada sera apprécié plus objectivement, puisque, sans négliger pour autant les traits de la survivance française, les témoignages mettront avant tout l'accent sur les facteurs économiques et sociaux.

(à suivre)

ARMAND YoN 